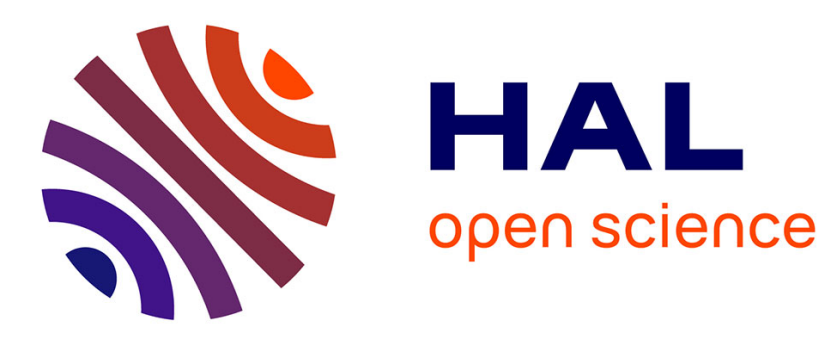

\title{
Decentralized constructive collision avoidance for Multi-Agent dynamical systems
}

Minh Tri Nguyen, Cristina Stoica Maniu, Sorin Olaru

\section{To cite this version:}

Minh Tri Nguyen, Cristina Stoica Maniu, Sorin Olaru. Decentralized constructive collision avoidance for Multi-Agent dynamical systems. 15th European Control Conference (ECC 2016), Jun 2016, Aalborg, Denmark. pp.1526-1531, 10.1109/ecc.2016.7810507 . hal-01390087

\section{HAL Id: hal-01390087 \\ https://hal.science/hal-01390087}

Submitted on 16 Mar 2020

HAL is a multi-disciplinary open access archive for the deposit and dissemination of scientific research documents, whether they are published or not. The documents may come from teaching and research institutions in France or abroad, or from public or private research centers.
L'archive ouverte pluridisciplinaire HAL, est destinée au dépôt et à la diffusion de documents scientifiques de niveau recherche, publiés ou non, émanant des établissements d'enseignement et de recherche français ou étrangers, des laboratoires publics ou privés. 


\title{
Decentralized Constructive Collision Avoidance for Multi-Agent Dynamical Systems
}

\author{
M.T. Nguyen, C. Stoica Maniu, S. Olaru
}

\begin{abstract}
This paper describes the principles of a decentralized framework for the guaranteed collision avoidance of Multi-Agent dynamical systems sharing a common working space. The results are constructive and can be effective in the certification of mission safety. The geometric aspects of the collision avoidance problem are exploited to define the control policies. The main contributions are related to the optimizationbased decentralized feedback control which renders a so-called functioning zone controlled invariant. An illustrative example is analyzed in order to highlight the effectiveness of the proposed approaches.
\end{abstract}

\section{INTRODUCTION}

Multi-Agent System (MAS) receives considerable attention due to the need to control a group of relatively independent sub-systems in order to achieve a common goal, such as traffic control, spacecraft formation, etc. Beside the performance quality, the mission safety is highly required. This criterion implies a supplementary fault diagnosis layer to detect and isolate possible faults at the agent level (e.g. actuator faults [1], sensor faults [2]) or at the formation level (e.g. communication faults [3]). Today, one of the most wellknown research field of MAS is the collision avoidance ([4], [5]) due to the need to avoid the damages caused from the collision with obstacles or with other agents.

Recently, set-theoretic and optimization tools are employed to design the control of MAS subject to anti-collision constraints, notable [6], [7], [8], [9] and [10]. In these works, the constraints are described such that the sets characterizing each agent/obstacle do not overlap. The authors of [8] show that regulating a single system subject to obstacle avoidance constraints is equivalent to control it around an equilibrium point outside of its domain of attraction. This can be also obtained by using adversary control [11]. In the context of MAS, a feedback control w.r.t the obstacle avoidance constraints for one agent is proposed in [12]. Other works on formation control are based on keeping a distance between the agents, e.g. sliding mode control [9] and model predictive control [7]. The recent work [10] proposes a set-theoretic method based on a decentralized control strategy.

In this context, the aim of the present paper is to propose a new decentralized approach to guarantee the collision avoidance using the leader-follower structure. The methodology will be described in $\mathbb{R}^{2}$ while the principles remain general. This work can be considered as a natural extension of the

M.T. Nguyen, C. Stoica Maniu and S. Olaru are with Laboratoire des Signaux et Systèmes, CentraleSupélec-CNRS-Univ. ParisSud, Université Paris Saclay, 3 rue Joliot Curie, F-91192, Gifsur-Yvette cedex, France (e-mail: \{minhtri.nguyen; cristina.stoica; sorin.olaru\}esupelec.fr). previous results [8] but applied for MAS and developed in a decentralized manner from the control computation point of view. We will analyze the restrictions of the method developed in [8], i.e. its feasibility limits and infeasible situations. One original aspect of the current paper is to extend the method used in [10] for the unbounded safety functioning zones and to propose a new approach to deal with both the feasible and the infeasible cases. Our main objective is to partition the working space into a collection of safety zones. Each of these zones is associated with only one agent and our novel control strategy will keep the agent operating in the interior of this zone, offering anti-collision guarantees.

The remainder of the paper is organized as follows. Section II briefly presents some useful prerequisites and further formulates the problem. Section III recalls the main ideas of the decentralized structure. The main contribution is described in Sections IV. Here, we analyze the cases leading to feasibility limitations or infeasibility of the decentralized control action for MAS and further propose new guaranteed solutions based on set-theoretic tools. Finally, numerical simulation results are illustrated in Section V, followed by concluding remarks and perspectives in Section VI.

Notations: $\mathcal{A} \oplus \mathcal{B}=\{a+b \mid a \in \mathcal{A}, b \in \mathcal{B}\}$ and $\mathcal{A} \ominus$ $\mathcal{B}=\{a \in \mathcal{A} \mid a+b \in \mathcal{A}, \forall b \in \mathcal{B}\}$ denote respectively the Minkovski sum and the Pontryagin difference of two sets $\mathcal{A}$ and $\mathcal{B} . \mathbb{N}_{[n, n+m]} \triangleq\{n, n+1, \ldots, n+m\} \subset \mathbb{N}$ for $n, m \geq 0$ is the set containing the natural numbers from $n$ to $n+m$, in increasing order. Consider a group of $N$ agents with the $i^{t h}$ agent being the leader. $\mathcal{F}^{i} \subseteq \mathbb{N}_{[1, N]} \backslash\{i\}$ is the set of indices of its followers identified by the existence of a connection to the node $i$. For the $j^{\text {th }}$ follower, its safety region is denoted by $\mathcal{S}_{j}$. Its functioning zone and its separation set are denoted respectively by $\mathcal{H}_{j}^{i}$ and $\mathcal{C}_{j}^{i}$ with respect to its leader $i$.

\section{PREReQuisites AND PROBlem FORMULATION}

This section presents firstly the theoretical background and secondly formulates the problem.

Definition 1. [13] Consider an autonomous discrete-time Linear Time Invariant (LTI) system $x^{+}=A x$, where $A$ is a Schur matrix. A set $\mathcal{S}$ is called positive invariant (PI) for this system, if $x^{+} \in \mathcal{S}$ for all $x \in \mathcal{S}$, which is equivalent to $A \mathcal{S} \subseteq \mathcal{S}$.

Definition 2. [8] Given the discrete-time LTI system dynamics $x^{+}=A x+B u$, a set $\mathcal{S} \in \mathbb{R}^{n}$ is linearly controlled invariant, if for any $x \in \mathcal{S}$ there exists a feedback control law $u=K x$ such that $(A+B K) x \in \mathcal{S}$. 
Theorem 1. [14] A set $\mathcal{S}=\left\{x \in \mathbb{R}^{n} \mid G x \leq \theta\right\}$ is positively invariant w.r.t the discrete-time LTI system dynamics $x^{+}=$ $A x$ if and only if there exists a matrix $H \in \mathbb{R}^{n \times n}$ with non-negative elements, such that $G A=H G$ and $H \theta \leq \theta$.

Definition 3. An ellipsoid $\mathcal{E}(c, d) \in \mathbb{R}^{n}$ with the center $c \in$ $\mathbb{R}^{n}$, the shape matrix $P \in \mathbb{R}^{n \times n}$ and the range $d \in \mathbb{R}$ is defined as $\mathcal{E}(c, d)=\left\{x \in \mathbb{R}^{n} \mid(x-c)^{\top} P(x-c) \leq d\right\}$, with $P=P^{\top} \succ 0$ and $d>0$.

Consider a Multi-Agent System $\boldsymbol{\Sigma}$ composed of $N$ agents. The nominal dynamics of each agent $i$ is:

$$
x_{i}^{+}=A_{i} x_{i}+B_{i} u_{i}, i \in \mathbb{N}_{[1, N]}
$$

where $x_{i} \in \mathbb{R}^{n}$ and $u_{i} \in \mathbb{R}^{m}$ are the $i^{t h}$ agent's nominal state and input vector. The pairs $\left(A_{i}, B_{i}\right)$ are assumed to be stabilizable. A convex bounded set $\mathcal{S}_{i}$ defined around the origin is considered as the safety region around the $i^{\text {th }}$ agent state ${ }^{1}$, i.e. $\mathcal{S}_{i}\left(x_{i}\right)=\left\{x_{i}\right\} \oplus \mathcal{S}_{i}$. The collision avoidance constraint between two agents can be described via the exclusion of their safety regions, i.e. $\mathcal{S}\left(x_{i}\right) \cap \mathcal{S}\left(x_{j}\right)=\emptyset$ yielding $\left(\left\{x_{i}\right\} \oplus \mathcal{S}_{i}\right) \cap\left(\left\{x_{j}\right\} \oplus \mathcal{S}_{j}\right)=\emptyset$, or equivalently $x_{j}-$ $x_{i} \notin\left(-\mathcal{S}_{j}\right) \oplus \mathcal{S}_{i}, \forall i \neq j$. In the centralized approaches, all of these constraints have to be strictly satisfied to guarantee the formation safety but it makes the MAS sensitive to communication faults. In order to overcome these drawbacks, a decentralized approach is proposed in the next section.

\section{Decentralized APproach}

The decentralized structure considered is the leaderfollower architecture [16]. In principle, an agent needs to be in relative formation and observe a number of constraints w.r.t its leader. It is assumed that each follower is connected uniquely to one leader which is the only one who possesses the complete information on its followers. The communication graphs are illustrated in Fig. 1 for the decentralized (the $1^{s t}$ agent is the leader) and the centralized approach.
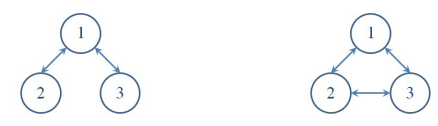

Fig. 1. Decentralized (left) and centralized (right) communication graph.

In this section, we will present firstly the leader-follower structure and further the control strategy used to ensure the collision avoidance. For brevity of the notation and presentation, the group of agents will be considered to evolve in $\mathbb{R}^{2}$ and the $i^{t h}$ agent will be considered as the leader.

\section{A. Leader-follower architecture}

The local closed-loop of the $j^{\text {th }}$ follower is $x_{j}^{+}=A_{j} x_{j}+$ $B_{j} u_{j}$, with its local control $u_{j}=\bar{u}_{j}^{i}+\mathcal{K}\left(x_{j}, \bar{x}_{j}^{i}\right)$. The pair $\left(\bar{x}_{j}^{i}, \bar{u}_{j}^{i}\right)$ represents the tracking reference of the $j^{\text {th }}$ follower, according to the current state and control action $\left(x_{i}, u_{i}\right)$ communicated by the leader. An offset free pair $\left(\bar{x}_{j}, \bar{u}_{j}\right)$ is

\footnotetext{
${ }^{1}$ The construction of $\mathcal{S}_{i}$ is presented in [15].
}

added to $\left(\bar{x}_{j}^{i}, \bar{u}_{j}^{i}\right)$ to avoid the collision with the leader, i.e. $\bar{x}_{j}^{i}=\bar{x}_{j}+x_{i}$ and $\bar{u}_{j}^{i}=\bar{u}_{j}+u_{i}$.

The collision avoidance is guaranteed if each follower operates strictly in its functioning zone relative to the position of the leader. This zone is defined by the leader and identified by a separating hyperplane for any pair of followers. Its construction is presented in the following subsection.

\section{B. Partitioning in functioning zones}

The leader uses a set of hyperplanes to separate the functioning zone of its followers, more precisely it partitions the working space into an union of polyhedral non-overlapping separation sets. Each set is associated to a follower and defines its functioning zone. For the $j^{\text {th }}$ follower, its separation set $\mathcal{C}_{j}^{i}$ is defined as a cone:

$$
\mathcal{C}_{j}^{i}=\left\{x \in \mathbb{R}^{n} \mid C^{i}\left(x-x_{i}\right) \leq 0, j \in \mathcal{F}^{i}\right\}
$$

where $C^{i}\left(x-x_{i}\right)=0$ denotes the set of hyperplanes used to isolate the $j^{\text {th }}$ follower from its neighbors.

Assumption 1. Instead of determining the functioning zone relative to the position of the leader, it can be translated without loss of generality to a positioning relative to the origin i.e. $\left(x_{i}, u_{i}\right)=(0,0)$.

Clearly, the hyperplanes pass through the leader current state, now reduced to the origin.

Remark 1. The choice of the separating hyperplanes $C^{i} x=$ 0 is important because it decides the existence of a feasible control action on the boundary of the separation set [10]. This choice is beyond the scope of the present work. In the remaining of the paper it is assumed that the hyperplanes $C^{i} x=0$ are given and the sets $\mathcal{C}_{j}^{i}$ are non-overlapping.

In order to ensure the collision avoidance, the state $x_{j}$ has to satisfy the two following constraints:

$$
\begin{aligned}
& x_{j} \notin \operatorname{Int}\left\{\left(-\mathcal{S}_{j}\right) \oplus \mathcal{S}_{i}\right\} \\
& \left\{x_{j}\right\} \oplus \mathcal{S}_{j} \in \mathcal{C}_{j}^{i}
\end{aligned}
$$

The constraint (3a) guarantees the collision avoidance between the $j^{\text {th }}$ follower with the leader. It is derived from $x_{j}-x_{i} \notin\left(-\mathcal{S}_{j}\right) \oplus \mathcal{S}_{i}$ with $x_{i}$ being the origin (see Assumption 1). The constraint (3b) guarantees the collision avoidance with the other followers. The $j^{\text {th }}$ follower with its safety region ${ }^{2}$ is the unique agent belonging to the separation set $\mathcal{C}_{j}^{i}$. This implies the anti-collision with its neighbors. These two constraints determine the non-convex functioning zone $\mathcal{Z}_{j}^{i} \subset \mathcal{C}_{j}^{i}$ in which $x_{j}$ has to be enclosed.

$$
\mathcal{Z}_{j}^{i}=\left\{x \in \mathbb{R}^{n} \mid\{x\} \oplus \mathcal{S}_{j} \in \mathcal{C}_{j}^{i}, x \notin \operatorname{Int}\left\{\left(-\mathcal{S}_{j}\right) \oplus \mathcal{S}_{i}\right\}\right\}
$$

\footnotetext{
${ }^{2}$ Note that, the effect of the possible disturbances is taken into account via the safety region $\mathcal{S}_{j}$. Practically, a hard constraint is imposed via (3b) for the center $x_{j}$ of the tube in steady functioning.
} 


\section{Local fixed point determination}

Starting from the characterization of the functioning zone sent by the leader, each follower has to find the best suitable equilibrium point relative to the origin (considered as the leader's state - Assumption 1) by solving its own optimization:

$$
\begin{array}{ll}
\bar{x}_{j}^{*}= & \arg \min _{\bar{u}_{j}}\left\|\bar{x}_{j}\right\| \\
\text { s.t. } & \bar{x}_{j} \in \mathcal{Z}_{j}^{i} \\
& \bar{x}_{j}=A_{j} \bar{x}_{j}+B_{j} \bar{u}_{j}
\end{array}
$$

The constraint (5b) implies that the non-convex set $\mathcal{Z}_{j}^{i}$ contains the local fixed point $\bar{x}_{j}$, as illustrated in Fig. 2 (with $i=1$ and $\left.\mathcal{F}^{i}=\{2,3\}\right)$. The constraint (5c) enforces the fixed point characterization.

In order to avoid the non-convexity of $\mathcal{Z}_{j}^{i}$, the constraint (5b) will be replaced for the local feedback design by $x_{j} \in$ $\mathcal{H}_{j}^{i}$, with $\mathcal{H}_{j}^{i} \subset \mathcal{Z}_{j}^{i}$ a convex region:

$$
\mathcal{H}_{j}^{i}=\left\{\mathcal{C}_{j}^{i} \ominus \mathcal{S}_{j}\right\} \cap\left\{h_{z} x \leq k_{z}\right\}
$$

with $\left\{h_{z} x=k_{z}\right\}$ the constraints activated when verifying the inclusion $\bar{x}_{j}^{*} \in\left\{\left(-\mathcal{S}_{j}\right) \oplus \mathcal{S}_{i}\right\}$. In Fig. 2 , the sets $\mathcal{Z}_{2}^{1}$ and $\mathcal{Z}_{3}^{1}$ are bounded by the black dash-line. The functioning zones $\mathcal{H}_{2}^{1}$ and $\mathcal{H}_{3}^{1}$ are green-covered and the fixed points $\left(\bar{x}_{2}^{*}, \bar{x}_{3}^{*}\right)$ found by solving (5) are plotted as the circle-red points. The green line represent the hyperplane used to separate the two followers, which in fact defines the separation sets $\mathcal{C}_{2}^{1}$ (on its left hand side) and $\mathcal{C}_{3}^{1}$ (on its right hand side). The forbidden blue set represents the anti-collision constraints $\left(-\mathcal{S}_{j}\right) \oplus \mathcal{S}_{i}$ between the leader and its followers (see (3a)).

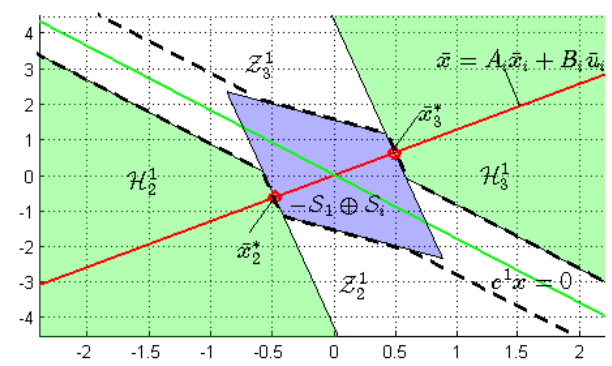

Fig. 2. Determination of $\mathcal{H}_{2}^{1}$ and $\mathcal{H}_{3}^{1}$ for the $2^{\text {nd }}$ and $3^{\text {rd }}$ agent.

Once the feasibility of the static optimization problem is guaranteed, each follower has to design the ego control policies to keep its own trajectories within its functioning zone and to asymptotically converge to the fixed point solution of (5). This control law will be developed in the next subsection.

\section{Individual feedback control}

In the sequel, for brevity, we will neglect the follower indices in the notations. The nominal local dynamics of a follower $x^{+}=A x+B u$, its functioning zone $\mathcal{H}$ and its fixed point $\bar{x}$ relative to the origin are considered known.

It will be considered that $\mathcal{H} \in \mathbb{R}^{2}$ is represented as the combination of linear inequalities $\mathcal{H}=\left\{x \in \mathbb{R}^{n} \mid h x \leq k\right\}$ similar to the one illustrated in the Fig. 2 where not all hyperplanes forming $\mathcal{H}$ are activated by $\bar{x}$ (as part of them are inherited from the functioning zone constraints). We denote $\left\{h_{1} x=k_{1}\right\}$ the set of hyperplanes activated by $\bar{x}$ and $\left\{h_{2} x=k_{2}\right\}$ the remaining hyperplanes.

The goal is to ensure that $x$ will converge asymptotically to a fixed point lying on the boundary $h_{1} x=k_{1}$, while the trajectory remains in $\mathcal{H}$ according to the constraints (3). This requires the design of a feedback linear $\operatorname{control}^{3}$ :

$$
u=K\left(h_{1}, k_{1}\right) x
$$

which makes the set $\mathcal{H}$ positive invariant and also stabilizes the closed-loop dynamics of the follower. According to [8], such a control law (7) exists if and only if there exist a non-negative matrix $F$ and matrix $P=P^{\top} \succ 0$ such that:

$$
\begin{aligned}
& h_{1}(A+B K)=F h_{1} \\
& {\left[\begin{array}{cc}
\delta P & (A+B K) P \\
P^{\top}(A+B K)^{\top} & P
\end{array}\right] \succeq 0}
\end{aligned}
$$

with the decision variables $F, K\left(h_{1}, k_{1}\right)$ and $P$. The constraint (8a) expresses the invariance condition (see Definition 2 and Theorem 1), while (8b) formulates the Lyapunov stability constraint, with $0<\delta<1$ the rate of convergence. However, solving this LMI (Linear Matrix Inequality) problem for $h_{1} x \leq k_{1}$ may face particular (infeasible) situations which will be discussed in the next section.

\section{MAIN CONTRIBUTION}

This section considers the two possibilities of solving (8):

1) Feasibility: A control $u=K\left(h_{1}, k_{1}\right) x$ which ensures the controlled invariance property of the domain described by the half-space active at $\bar{x}$, i.e. $h_{1} x \leq k_{1}$ is obtained. What remains to be considered is the satisfaction of the inactive constraints at $\bar{x}$, i.e. $h_{2} x \leq k_{2}$, or equivalently, the controlled invariance of this domain w.r.t the control $u$.

2) Infeasibility: The set $\mathcal{H}$ is not controlled invariant even if the closed-loop dynamics is stabilized. In this case, one solves the LMI problem for a strict subset of $\mathcal{H}$.

Both of these two cases were not completely analyzed in the literature. The main contribution of the present paper is to employ set-theoretic tools in order to analyze and to propose novel control strategies to deal with both the feasible and the infeasible cases. Two approaches are provided for the feasible case in the subsection IV-A. A relaxed construction is proposed for the infeasible case in the subsection IV-B.

\section{A. Feasible fixed point $\bar{x}$}

If the optimization problem (8) is feasible, then there exists a control $u=K\left(h_{1}, k_{1}\right) x$ ensuring the invariance of the subspace $h_{1} x \leq k_{1}$. If $u$ can not guarantee the invariance of the entire set $\mathcal{H}$, then there exists an invariant subset $\Omega \subseteq \mathcal{H}$.

The set $\Omega$ can be characterized in terms of the Maximal Output Admissible Set (MOAS) [17] associated with the

\footnotetext{
${ }^{3}$ If $k_{1} \neq 0$, this control is affine, i.e. $u=K\left(h_{1}, k_{1}\right) x+l\left(h_{1}, k_{1}\right)$, with $l\left(h_{1}, k_{1}\right)$ representing the affine term. In order to simplify the presentation of the proposed results, we will consider here the homogeneous case.
} 
local control $u_{\Omega}=K\left(h_{1}, k_{1}\right) x$. Knowing that $\Omega \subseteq \mathcal{H}$ and $A+B K\left(h_{1}, k_{1}\right)$ is a Schur matrix, there exist constructive methods to obtain iteratively $\Omega$ as proposed in [17]. We denote $\Omega(t)$ the MOAS obtained at each iteration $t$, with $\Omega(t)=\left\{x \in \mathbb{R}^{n} \mid h_{\Omega} x \leq k_{\Omega}\right\}$ and $\Omega(t) \subset \mathcal{H}$.

Supposing there exists a finite time $t \geq t^{*}$ such that $\Omega(t+1)=\Omega(t)=\Omega$, then the finite determinedness can be guaranteed and further exploited. This allows a construction as the one illustrated in Fig. 3, starting from $\Omega(0)=\mathcal{H}$ (bounded by the dash line) and contracting to $\Omega$ (darkest blue colored $-\Omega=\Omega(3)$ in this case).

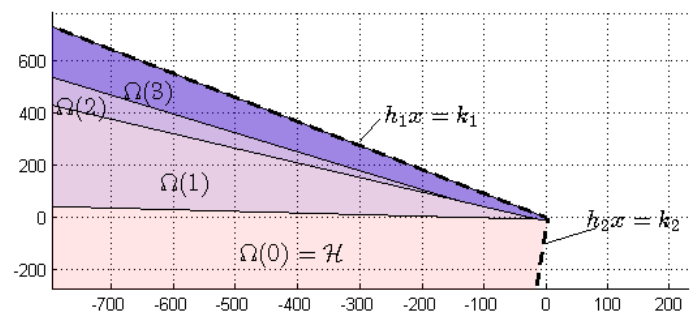

Fig. 3. Construction of $\Omega$

Remark 2. The control law $u_{\Omega}=K\left(h_{1}, k_{1}\right) x$ makes the entire half-space $h_{1} x \leq k_{1}$ controlled invariant. But $\mathcal{H}$ is a subset of this half-space and has $h_{1} x \leq k_{1}$ as its boundary. It follows that one of the boundary of $\Omega$ is $h_{1} x=k_{1}$.

Remark 3. If $\mathcal{H}$ is controlled invariant by $u_{\Omega}$ then $\Omega=\mathcal{H}$.

We note that the local control is restricted in the subset $\Omega$, and it will be activated only when the current state belongs to $\Omega$. Whenever $x \notin \Omega$, the linear control law is not admissible. One has to devise a procedure to drive the agent state $x$ into the strict interior of $\Omega$ while ensuring the invariance of $\mathcal{H}$.

We propose here two approaches to treat this problem. The first one, based on the iterative construction of a set of contracted ellipsoids inside of $\mathcal{H}$, is the main novelty of this paper. The second approach employs the interpolation technique for the case of unbounded functioning zones.

1) Approach 1 - Parameterized contractive ellipsoid: This approach uses the existence of an ellipsoid (see Definition 3 ) in the functioning zone to drive the current state into the set $\Omega$. It takes the form of an optimization problem (9) with a given shape of the ellipsoidal set, while the center and the range are considered as parameters. Consider the explicit form of the functioning zone $\mathcal{H}=$ $\left\{a_{l} x \leq b_{l}, \forall l \in \mathbb{N}_{[1, \ldots p]}\right\}$, with $p$ denoting the number of constraints, which leads to:

$$
\begin{array}{ll}
c^{*}(x)=\arg \min _{c, d, u_{c}} \log (|c-\bar{x}|) \\
\text { s.t. } \quad c=A c+B u_{c} \\
& \left(\sqrt{a_{l} P^{-1} a_{l}^{\top}}\right) \sqrt{d}+a_{l} c \leq b_{l} \\
& (x-c)^{\top} P(x-c) \leq d
\end{array}
$$

The constraint (9b) implies that the center $c$ is a fixed point. The shape matrix $P$ is obtained by solving (8) for $h_{1} x \leq k_{1}$. The constraints (9c) and (9d) imply respectively that the ellipsoid is inside of $\mathcal{H}$ and contains the current state vector $x$. We explain further how to determine these constraints.

Theorem 2. For a given $x$, if (9) is feasible, then there exists an ellipsoid included inside of $\mathcal{H}$ and $x^{+} \in \mathcal{H}$.

Proof. The optimization problem corresponds to an inclusion of an ellipsoid in a polyhedral set [18]. We recall that feasibility of (8) for the half-space $h_{1} x \leq k_{1}$ implies the existence of an ellipsoid $\mathcal{E}(\bar{x}, 1)=\left\{x \in \mathbb{R}^{n} \mid(x-\bar{x})^{\top} P(x-\bar{x}) \leq 1\right\}$. We want to check if by using $u=K\left(h_{1}, k_{1}\right) x$, the constraint $x^{+} \in \mathcal{H}$ holds for every $x \in \mathcal{H}$. This can be translated to finding an ellipsoid included in the strict interior of $\mathcal{H}$ which satisfies: (i) the current state is included in the ellipsoid (9d), (ii) it preserves the shape of $P$ and optimizes its offset and scaling, (iii) its center $c=c(x)$ is situated on the fixed point hyperplane (9b), (iv) $c$ is as close as possible to $\bar{x}$. This ellipsoid has the parameterized form $\mathcal{E}(c, d)=\left\{x \in \mathbb{R}^{n} \mid(x-c)^{\top} P(x-c) \leq d\right\}$. Consider $y=\left(\frac{P}{d}\right)^{1 / 2}(x-c)$ and denote the norm operation $\|q\|=$ $\sqrt{q^{\top} q}$ for some vector $q$. The ellipsoid form becomes $\mathcal{E}(c, d)=\left\{\left(\frac{P}{d}\right)^{-1 / 2} y+c \in \mathbb{R}^{n} \mid\|y\| \leq 1\right\}$. Considering $\mathcal{E}(c, d) \subseteq \mathcal{H}$, then for all $l \in \mathbb{N}_{[1, \ldots p]}$ and $\|y\| \leq 1$, we derive $a_{l}\left(\left(\frac{P}{d}\right)^{-1 / 2} y+c\right) \leq b_{l}$ and so $\left\|\left(\frac{P}{d}\right)^{-1 / 2} a_{l}^{\top}\right\|+a_{l} c \leq b_{l}$. Hence we get $\left(\sqrt{a_{l} P^{-1} a_{l}^{\top}}\right) \sqrt{d}+a_{l} c \leq b_{l}$. Whenever $\mathcal{E}(c, d)$ exists, by the inclusion of $\mathcal{E}(c, d)$ in the strict interior of $\mathcal{H}$, one can guarantee the stability of the closed-loop, and the recursive constraint satisfaction as long as $x^{+} \in \mathcal{H}$.

For a given $x$, its convergence to the fixed point $\bar{x}$ is guaranteed by minimizing the distance between $c$ and $\bar{x}$. This optimization problem is repeated until the state trajectory reaches $\Omega$ as long as the linear feedback law is known to be feasible in $\Omega \subset \mathcal{H}$. An illustration is shown in Fig. 4. The green ellipsoid $\mathcal{E}(\bar{x}, 1)$ is obtained according to the solution $P$ of (8), centered in $\bar{x}$ (red circle). The red line represents the set of fixed points and the blue circle points are the centers of the ellipsoids obtained by solving iteratively (9) according to the feedback state $x$ (black plus sign). We can see that $x$ approaches $\bar{x}$ according to the convergence of $c(x)$ into $\bar{x}$.

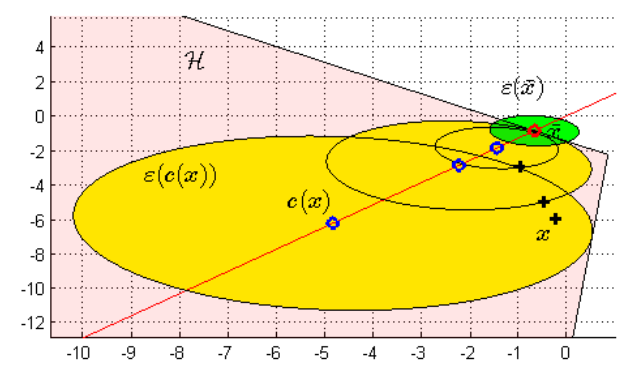

Fig. 4. Feasibility test 
Remark 4. The square root terms make (9) nonlinear. We can avoid its complexity in $(9 c)$ by replacing $\sqrt{d}$ by the new variable $\theta$, i.e. $\left(\sqrt{a_{l} P^{-1} a_{l}^{\top}}\right) \theta+a_{l} c \leq b_{l}$. Then, we can subsequently use the parameter $\epsilon$ to decrease the gap between the variables $d$ and $\theta$, i.e. $0 \leq \theta^{\top} \theta-d \leq \epsilon$, and thus transform (9) into a classical convex optimization problem.

Remark 5. The problem (9) is not guaranteed to cover the maximal controllable subset of $\mathcal{H}$. For initial points on the vertices of $\mathcal{H}$ the feasibility of the ellipsoidal containment cannot be fulfilled as long as there are two different supporting hyperplanes active for the same point on the boundary.

In the light of Remark 5, we propose a second approach which deals with the infeasibility of (9) for the current $x$.

2) Approach 2 - Interpolation based control: The control action $u$ will be obtained as a convex combination of the local control $u_{\Omega}=K\left(h_{1}, k_{1}\right) x$ in the MOAS $\Omega$ and a vertex control law ${ }^{4}$ associated with a M-step Robustly Controlled Set $\mathcal{P}_{M}$. The principles of such an interpolation based control were discussed in [19] and applied in [10]. The feasible region for the interpolation scheme will be restricted to the maximal controllable set inside $\mathcal{H}$. This set can be obtained using classical reachability arguments (see [13] for details).

The present work consideres that either the set $\mathcal{P}_{M}$ or a convex (polyhedral) controlled invariant subset $\Phi$ (such as $\Omega \subseteq \Phi \subseteq \mathcal{P}_{M}$ ) is available together with a feasible control action on the boundaries (the so-called vertex control [20]). Therefore, the interpolated control action becomes $u=\beta u_{\Phi}+(1-\beta) u_{\Omega}, 0 \leq \beta \leq 1$, where $u_{\Phi}$ and $\beta$ have to be calculated in real time. The control component $u_{\Phi}$ will be activated when $x \in(\Phi \backslash \Omega)$ and the scalar $\beta$ has to be minimized in order to get $u$ as close as possible to $u_{\Omega}$.

For simplicity consider the set $\Phi$ to be a scaled outer version of $\Omega$ with the restriction to $\mathcal{H}$. The scaling of the set $\Omega$ will be done with respect to the fixed point $\bar{x}$.

Proposition 1. An outer candidate set for the interpolation $\Phi$ is defined as $\Phi=\left(\{\bar{x}\} \oplus \mu \Omega_{0}\right) \cap \mathcal{H}$, with $\mu$ found by solving the following linear programming $(L P)$ problem:

$$
\min _{\mu} \mu \text { s.t. }\left\{\begin{array}{l}
x \in \mu \Omega_{0} \\
\mu \geq 0
\end{array}\right.
$$

with $\Omega_{0}=\{-\bar{x}\} \oplus \Omega$.

Remark 6. The set $\Phi$, obtained via the optimal solution for the LP problem (10) ensures $\Omega \subseteq \Phi$.

Consider an example in Fig. 5. The set $\mathcal{H}$ is bounded by $h_{1} x=k_{1}$ and $h_{2} x=k_{2}$ (dash line). The fixed point (red) is on the boundary $h_{1} x=k_{1}$. The orange set is the MOAS $\Omega$. The set $\Phi$ (with $\Omega \subset \Phi \subset \mathcal{H}$ ) is colored in yellow.

We present next how to effectively obtain the vertex control action $u_{\Phi}$ and then the interpolation coefficient $\beta$.

The determination of $u_{\Phi}$ exploits the fact that its objective is to push $x$ from the boundary of $\Phi$ towards its interior. This

\footnotetext{
${ }^{4} \mathrm{~A}$ vertex control for a given polyhedral state-space set is defined as a control action used to drive a current state from the corresponding vertex toward the interior of the considered polyhedron.
}

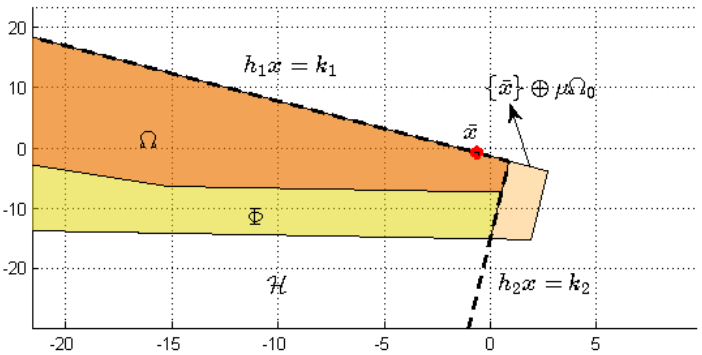

Fig. 5. Construction of the interpolation set $\Phi$

can be done by solving the following LP problem:

$$
u_{\Phi}=\arg \min _{\alpha, u_{\Phi}} \alpha \text { s.t. }\left\{\begin{array}{l}
A x+B u_{\Phi} \in \alpha \Phi \\
0 \leq \alpha \leq 1
\end{array}\right.
$$

with $\alpha$ denoting the minimal contraction factor.

For the determination of the scalar $\beta$, we exploit the fact that the control $u$ obtained as an interpolation of $u_{\Omega}$ and $u_{\Phi}$ has to be as close as possible to $u_{\Omega}$. This can be translated in terms of the optimization problem. Consider $u=\beta u_{\Phi}+$ $(1-\beta) u_{\Omega}$, and $x$ decomposed as $x=\beta x_{\Phi}+(1-\beta) x_{\Omega}$, where $x_{\Phi} \in \Phi$ and $x_{\Omega} \in \Omega$. Let $\Phi=\left\{x \mid F_{\Phi} x \leq k_{\Phi}\right\}$ and $\Omega=\left\{x \mid F_{\Omega} x \leq k_{\Omega}\right\}$. Then the optimization problem is

$$
\min _{\beta, x_{\Omega}, x_{\Phi}} \beta \text { s.t. }\left\{\begin{array}{l}
F_{\Phi} x_{\Phi} \leq k_{\Phi} \\
F_{\Omega} x_{\Omega} \leq k_{\Omega} \\
\beta x_{\Phi}+(1-\beta) x_{\Omega}=x \\
0 \leq \beta \leq 1
\end{array}\right.
$$

Although (12) is nonlinear, we can translate it into a LP problem by using $r=\beta x_{\Phi}$. Thus (12) becomes:

$$
\min _{\beta, r} \beta \quad \text { subject to }\left\{\begin{array}{l}
F_{\Phi} r \leq \beta k_{\Phi} \\
F_{\Omega}(x-r) \leq(1-\beta) k_{\Omega} \\
0 \leq \beta \leq 1
\end{array}\right.
$$

Solving respectively (10), (11), (13) in real time provides the interpolation factor and implicitly the control $u$.

\section{B. Infeasible fixed point $\bar{x}$}

In this case, the local control $u_{\Omega}=K\left(h_{1}, k_{1}\right) x$ can not guarantee the invariance of the half-space $h_{1} x \leq k_{1}$ and implicitly of the set $\mathcal{H}$ as long as the fixed point is placed on the boundary. Therefore, the local set $\Omega$ can not be constructed and the interpolation based control can not be used. In this case one can always find a subset in the strict interior of $\mathcal{H}$ but we have to deal with the nonlinear nature of (8). Indeed, the authors in [8] mentioned that only a subclass of design problem can be solved by eigenstructure assignment approaches. Here we propose a simpler and efficient method to solve this problem: by moving the fixed point $\bar{x}$ into the strict interior of $\mathcal{H}$. Once this relaxation is performed, any linear stabilizing feedback gain is admissible. Subsequently, the ellipsoidal construction approach presented above can be used to drive the current state $x$ according to the ellipsoid whose center is parametrized to enable the feasibility. This construction ensures the recursive feasibility and approached the fixed point arbitrarily close to the boundary of $\mathcal{H}$. 


\section{ILlustrative EXAMPLE}

Consider a MAS $\boldsymbol{\Sigma}$ composed of $N=3$ agents. They have the same safety region. Their dynamics is $x_{i}(k+1)=$ $\left[\begin{array}{cc}-0.2 & 0.5 \\ 0.2 & 0.71\end{array}\right] x_{i}(k)+\left[\begin{array}{l}0.71 \\ 0.22\end{array}\right] u_{i}(k)$. Let us choose the $1^{s t}$ agent as the leader. We will study the local feedback gain of the $2^{\text {nd }}$ agent (similar for the $3^{\text {rd }}$ agent). Two different initial points $x_{2}(0)=\left[\begin{array}{ll}-1 & -5\end{array}\right]^{\top}$ and $x_{2}(0)=\left[\begin{array}{ll}0 & -14.1643\end{array}\right]^{\top}$ are considered. As shown in Fig. 6, the first initial point

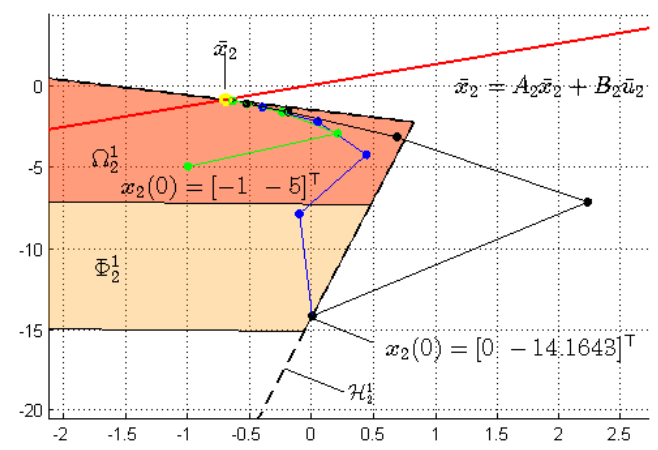

Fig. 6. Evolution of the $2^{\text {nd }}$ agent from different initial points.

$x_{2}(0)=\left[\begin{array}{ll}-1 & -5\end{array}\right]^{\top}$ is already included in $\Omega_{2}^{1}$. Hence both the parameterized contractive ellipsoid approach and the interpolation based control approach can be applied. In the first approach, the optimization problem (9) is successfully solved at each iteration, providing all of the ellipsoids centers to which $x_{2}$ converges (circle green line). Using the second approach, the local control $u_{\Omega_{2}}$ is activated. The state $x_{2}$ reaches the fixed point (circle green line). The other initial point $x_{2}(0)=\left[\begin{array}{ll}0 & -14.1643\end{array}\right]^{\top}$ situated on one boundary of $\mathcal{H}_{2}^{1}$ but not included in $\Omega_{2}^{1}$ cannot use the local linear control action. Moreover this boundary is not activated by the fixed point. We see that the control $u_{\Omega_{2}}$ can not ensure the control invariance of $\mathcal{H}_{2}^{1}$ (bounded by the dash line) even if $x_{2}$ still converges to the fixed point (circle black line). This choice of $x_{2}(0)$ in the inactive boundary implies that the ellipsoid approach can not be applied, because there do not exist any ellipsoid which contains $x_{2}(0)$ and which is included in the functioning zone $\mathcal{H}_{2}^{1}$. The interpolation approach is then employed and $x_{2}$ is kept in the interior of $\Omega_{2}$ (circle blue line).

\section{CONCLUSION}

This paper presents a decentralized approach to deal with the collision avoidance of Multi-Agent Systems. The main tool employed is the optimization-based control design with the goal of enforcing the control invariance of the safety functioning zone. The main advantage is that the local feasibility can be handled, which is considered novel compared to set-theoretic based decentralized control in the literature. We consider the cases when there exists a local control action to keep each agent in its strict functioning zone. The feasibility of this determination however is limited in a subset of the functioning zone, assimilated to a controlled invariant set via linear feedback. For the points which are not covered by this set, we propose a control strategy to drive the agent state towards the region where the local linear control action is feasible. Future work will focus on the construction of the set of hyperplanes used to separate the followers. The choice of these hyperplanes is important because they impact the feasibility of the control action.

\section{REFERENCES}

[1] G. Franze, F. Tedesco, and D. Famularo, "Actuator fault tolerant control: a set-theoretic approach," in IEEE Conference on Decision and Control, Hawaii, USA, 2012.

[2] F. Stoican, S. Olaru, and G. Bitsoris, "A fault detection scheme based on controlled invariant sets for multisensor systems," in Conference on Control and Fault Tolerant Systems, Nice, France, 2010.

[3] N. Meskin, K. Khorasani, and C. A. Rabbath, "A hybrid fault detection and isolation strategy for a network of unmanned vehicles in presence of large environmental disturbances," Control Systems Technology, IEEE Transactions on, vol. 18, no. 6, pp. 1422-1429, 2010.

[4] H. G. Tanner, A. Jadbabaie, and G. J. Pappas, "Flocking in teams of nonholonomic agents," in Cooperative Control. Springer, 2005, pp. 229-239.

[5] O. Khatib, "Real-time obstacle avoidance for manipulators and mobile robots," The international journal of robotics research, vol. 5, no. 1, pp. 90-98, 1986.

[6] I. Prodan, "Commande sous contraintes de systemes dynamiques multi-agents," Ph.D. thesis, Supelec (in English), 2012.

[7] A. Bemporad and C. Rocchi, "Decentralized linear time-varying model predictive control of a formation of unmanned aerial vehicles," in 50th IEEE Conference on Decision and Control and European Control Conference, 2011, pp. 7488-7493.

[8] G. Bitsoris and S. Olaru, "Further results on the linear constrained regulation problem," in Mediterranean Control Conference, Greece, June 2013.

[9] R. Bencatel, M. Faied, J. Sousa, and A. R. Girard, "Formation control with collision avoidance," in 50th IEEE Conference on Decision and Control and European Control Conference, 2011, pp. 591-596.

[10] M. T. Nguyen, C. Stoica Maniu, and S. Olaru, "Control invariant partition for heterogeneous multi-agent dynamical systems," in $19^{\text {th }}$ International Conference on System Theory, Control and Computing, Cheile Gradistei, Romania, 2015.

[11] E. Kontouras, A. Tzes, and L. Dritsas, "Adversary control strategies for discrete-time systems," in European Control Conference, 2014, pp. 2508-2513.

[12] I. Prodan, G. Bitsoris, S. Olaru, C. Stoica, and S.-I. Niculescu, "Constrained control design for linear systems with geometric adversary constraints," in IFAC $5^{\text {th }}$ Symposium on System Structure and Control, Grenoble, vol. 5, no. 1, 2013, pp. 815-820.

[13] F. Blanchini and S. Miani, Set-theoretic methods in control. Birkhauser, 2007.

[14] G. Bitsoris, "On the positive invariance of polyhedral sets for discretetime systems," Systems \& Control Letters, vol. 11, no. 3, 1988.

[15] D. Q. Mayne, M. M. Seron, and S. Raković, "Robust model predictive control of constrained linear systems with bounded disturbances," Automatica, vol. 41, no. 2, pp. 219-224, 2005.

[16] S. Stankovic, M. Stanojevic, and D. Siljak, "Decentralized overlapping control of a platoon of vehicles," Control Systems Technology, IEEE Transactions on, vol. 8, no. 5, pp. 816-832, Sep 2000.

[17] E. Gilbert and K. T. Tan, "Linear systems with state and control constraints: the theory and application of maximal output admissible sets," Automatic Control, IEEE Transactions on, vol. 36, no. 9, Sep 1991.

[18] H. Hindi, "A tutorial on convex optimization," in IEEE American Control Conference, vol. 4, June 2004, pp. 3252-3265.

[19] H.-N. Nguyen, P.-O. Gutman, S. Olaru, and M. Hovd, "Implicit improved vertex control for uncertain, time-varying linear discretetime systems with state and control constraints," Automatica, vol. 49, no. 9, pp. $2754-2759,2013$.

[20] P.-O. Gutman and M. Cwikel, "Admissible sets and feedback control for discrete-time linear dynamical systems with bounded controls and states," IEEE TAC, vol. 31, no. 4, pp. 373-376, 1986. 